\title{
Electronic Health Self-Management Interventions for Patients With Chronic Kidney Disease: Systematic Review of Quantitative and Qualitative Evidence
}

Hongxia Shen ${ }^{1}$, MSc; Rianne M J J van der Kleij ${ }^{1,2}$, PhD; Paul J M van der Boog ${ }^{3}$, MD, PhD; Xinwei Chang ${ }^{4}$, MSc; Niels H Chavannes ${ }^{1}, \mathrm{MD}, \mathrm{PhD}$

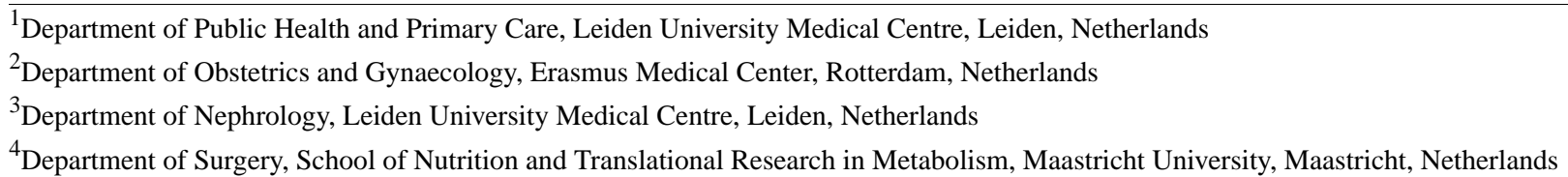

\section{Corresponding Author:}

Hongxia Shen, MSc

Department of Public Health and Primary Care

Leiden University Medical Centre

Albinusdreef 2

Leiden, $2333 \mathrm{ZA}$

Netherlands

Phone: 31633789207

Email: H.Shen@lumc.nl

\section{Abstract}

Background: Chronic kidney disease (CKD) poses a major challenge to public health. In CKD patients, adequate disease self-management has been shown to improve both proximal and distal outcomes. Currently, electronic health (eHealth) interventions are increasingly used to optimize patients' self-management skills.

Objective: This study aimed to systematically review the existing evidence regarding the implementation and effectiveness of eHealth self-management interventions for patients with CKD.

Methods: Following a search in 8 databases (up to November 2017), quantitative and qualitative data on process and effect outcomes were extracted from relevant studies. Quality was appraised using the Crowe Critical Appraisal Tool; narrative synthesis was performed to analyze the data extracted.

Results: Of the 3307 articles retrieved, 24 (comprising 23 studies) were included in this review; of these, almost half were appraised to be of low to moderate quality. There was considerable heterogeneity in the types of interventions used and the outcomes measured. A total of 10 effect and 9 process outcome indicators were identified. The most frequently reported effect outcome indicators were specific laboratory tests and blood pressure (BP), whereas satisfaction was the most frequently reported process outcome indicator. Positive effects were found for proximal outcomes (eg, BP control and medication adherence), and mixed effects were found for more distal outcomes (eg, quality of life). High feasibility, usability, and acceptability of and satisfaction with eHealth self-management interventions were reported. The determinant ability of health care professionals to monitor and, if necessary, anticipate on patient measurements online was mostly cited to influence patients' adherence to interventions.

Conclusions: eHealth self-management interventions have the potential to improve disease management and health outcomes. To broaden the evidence base and facilitate intervention upscaling, more detailed descriptions and thorough analysis of the intervention components used are required. In addition, our review reveals that outcomes closely related to the scope and duration of the intervention implemented are most likely to be impacted. For instance, if a 4-week Web-based training to optimize disease management skills is implemented, the outcome perceived control would more likely be affected than kidney function. Although this seems obvious, most studies evaluate only distal outcomes and thereby fail to capture intervention effects that might contribute to long-term health improvement. We advise future researchers to carefully consider their choice of outcomes based on their sensitivity for change. In this way, we ensure that relevant effects are captured and legitimate conclusions are drawn. 
(J Med Internet Res 2019;21(11):e12384) doi: 10.2196/12384

\section{KEYWORDS}

eHealth; self-management; systematic review; chronic kidney disease

\section{Introduction}

\section{Background}

Chronic kidney disease (CKD) is a major public health concern [1-3]. Globally, more than 70 million individuals are affected by CKD [4]. CKD is defined as kidney damage or a measured glomerular filtration rate (GFR) of $\leq 60 \mathrm{~mL} / \mathrm{min} / 1.73 \mathrm{~m}^{2}$ for more than 3 months. CKD is classified into 5 stages based on GFR decline [5]. The level of kidney function deterioration has a direct relationship with an increase in morbidity and mortality [6], poorer patient outcomes [3], higher hospitalization rates [7], and substantial increase in health care expenditures [8]. Patients with CKD report a lower quality of life (QoL) [9] and may experience severe medical complications and cognitive dysfunction [10].

Disease self-management (hereafter referred to as self-management) is defined as "an individual's ability to manage the symptoms, treatment, physical and psychosocial consequences, and lifestyle changes inherent to the life with a chronic condition" [11]. Adequate self-management is reported to improve patients' health behaviors targeted by the intervention (ie, proximal outcomes) and also indirect outcomes, such as disease characteristics and progress (ie, distal outcomes) [12-14]. Although the potential benefits of self-management interventions are widely reported in the literature, extrapolating these results in day-to-day practice is difficult. Lack of efficacy in practice might be related to a suboptimal implementation of the self-management interventions [15,16]. Reported barriers were often related to intervention characteristics, such as lack of tailoring to the individual patient. Moreover, a lack of patient involvement in intervention design and insufficient care continuity and accessibility were reported to hamper implementations $[17,18]$.

Electronic health (eHealth) technologies can help address implementation barriers by making interventions more accessible, acceptable, tailored, and interactive [19-21]. The most cited definition of eHealth is that by Eysenbach [22]:

\section{e-health is [...] referring to health services and information delivered or enhanced through the Internet and related technologies. In a broader sense, characterizes [...] to improve health care locally, regionally, and worldwide by using information and communication technology.}

eHealth can help patients achieve personal health goals, and it allows them to feel more responsible for their health status [23]. Moreover, eHealth facilitates remote patient communication and exchange of health data, helping to increase health care efficiency while maintaining a wide-scale, cost-effective health care approach [24]. eHealth interventions have been successfully implemented to support weight loss [25,26], promote smoking cessation [27], reduce depressive symptoms [28], and decrease mortality rates and acute admissions [29]. In addition,
eHealth-based interventions have been successfully applied to manage chronic disease [30-32].

Several studies have reported the use of eHealth-based self-management interventions in CKD [33-36]. Moreover, 3 systematic reviews were published on this topic [37-39]. However, these reviews only concentrated on 1 particular eHealth application, such as telemedicine; dietary mobile apps; and automated information technology tools. Moreover, these reviews focused on a limited number of study designs and outcomes. For example, 2 reviews only included randomized controlled trials (RCTs) $[38,39]$, and 1 review excluded studies focusing on implementation outcomes such as feasibility, validity, and acceptability [39]. Moreover, none of these reviews [37-39] reviewed the contribution of individual intervention components (eg, self-monitoring) to the effects found. These limitations of previous reviews make it difficult for researchers and intervention developers to determine which components should be employed to maximize the effectivity of eHealth self-management interventions for CKD patients.

\section{Objectives}

This study, therefore, aimed to systematically review the available evidence on eHealth-based self-management interventions for CKD. In specific, we aimed to review the following: (1) study characteristics and type of eHealth applications used; (2) intervention components implemented and, if possible, their relative contribution to the effect found; (3) both process and effect outcomes; and (4) determinants of implementation.

\section{Methods}

\section{Protocol and Registration}

This review was performed according to the Preferred Reporting Items for Systematic Reviews and Meta-Analyses (PRISMA) statement [40]. The protocol was registered in the international Prospective Register of Systematic Reviews database (Centre for Reviews and Dissemination [CRD] number: CRD 420180 81681).

\section{Search Methodology}

A systematic search was conducted to identify relevant articles; the search strategy was developed in collaboration with a certified librarian. In total, 8 electronic databases (PubMed, EMBASE, Web of Science, Cochrane Library, EmCare, PsycINFO, Academic Search Premier, and Science Direct) were searched in November 2017. Search terms covered 3 areas: (1) CKD, (2) eHealth, and (3) self-management (see Multimedia Appendix 1). Reference lists of the included studies were searched to identify other relevant articles. EndNote X9 (Clarivate Analytics) was used to support the review process. 


\section{Eligibility Criteria}

Inclusion and exclusion criteria (Textbox 1) were determined using the Patients, Interventions, Comparison, Outcomes, Study design methodology [41].

Textbox 1. Inclusion and exclusion criteria for this study.

Inclusion criteria:

- $\quad$ Participants - patients classified with chronic kidney disease (stage 1-5)

- Intervention-eHealth technologies ("any information and communication technology designed to deliver or enhance health services and information") applied to facilitate chronic kidney disease patients' self-management ("the care taken by individuals towards their own health and well-being: it comprises the actions they take to lead a healthy lifestyle; to meet their social, emotional and psychological needs; to care for their long-term condition, and to prevent further illness or accidents") [11]

- Comparison-no restrictions

- Outcomes - articles reporting on clinical (ie, patients' intermediate outcomes or clinical parameters of disease severity, such as blood pressure, fluid management, and mortality), humanistic (ie, consequences of disease or treatment on patients' functional status or quality of life, such as physical functioning, well-being, and levels of depression or anxiety), economic and utilization (ie, measures of health resource utilization, medical costs, and cost-effectiveness), and/or process (ie, indicators that affect patient care by improving health care delivery or patient-health care interactions and self-management related-factors, such as adherence to intervention, usability of eHealth technologies, and self-efficacy) outcomes

- Language restrictions_-articles needed to be written in English

- Study design—randomized and nonrandomized controlled trials, noncomparative trials, and qualitative or mixed methods articles

Exclusion criteria:

- $\quad$ Type of electronic health used-studies with devices only used for communication (eg, a telephone only used for a follow-up call) or data collection (eg, an internet system solely used to collect patient data without further intervention) purposes

- $\quad$ Study design — case reports containing $\leq 3$ participants, commentaries, reviews, letters, dissertations, editorials, conference proceeding, and books

\section{Study Identification}

After removal of duplications, titles and abstracts of the retrieved articles were screened independently by 2 reviewers (HS and $\mathrm{XC}$ ). Articles that did not meet inclusion criteria were removed. Potentially relevant articles were obtained in full text and reviewed independently by 2 authors (HS and XC). Any disagreements between the 2 authors were resolved by consensus or consultation with a third author (RK).

\section{Data Collection}

Data collection was performed independently by 2 reviewers (HS and XC) using a standardized data extraction form. Study characteristics, descriptions of eHealth self-management interventions (eg, intervention components), process and effect outcome indicators, and determinants of implementation were extracted. Discrepancies in extraction were discussed until consensus was reached.

\section{Quality Assessment}

Article quality was appraised independently by HS and XC using the Crowe Critical Appraisal Tool (CCAT) [42]—a reliable, widely used quality appraisal tool $[43,44]$. Use of the CCAT user guide promoted validity and inter-rater reliability [43-46]. The CCAT form is divided into 8 categories and 22 items, with a total of 99 subitems. Subitems are rated on a scale of present, absent, or not applicable. A 6-point scale ranging from 0 (the lowest) to 5 (the highest) is used to assign score per category, with 40 being the maximum achievable total score.

The CCAT does not allow for a qualitative comparison of appraisal scores. Hence, we used the star score system developed by our research group to compare study quality [47]. First, we calculated a quality score based on the CCAT. Then, a mean score and standard deviation of the quality scores were calculated. Star scores were then assigned to each article: 1 star if a quality score was more than $1 \mathrm{SD}$ below mean; 2 stars if a quality score ranged from $1 \mathrm{SD}$ below mean to mean score. The kappa between the 2 reviewers' scores of quality assessment was 0.63 , reflecting substantial agreement [48].

\section{Data Synthesis}

Data were reviewed using narrative synthesis [49]. Study characteristics were reviewed, summarized, and analyzed in a spreadsheet. In accordance with previous categorizations of eHealth $[32,39,50]$, eHealth self-management interventions were split into 5 major types (see Multimedia Appendix 2). eHealth functionalities used were described based on the technology functionality framework [51,52]. In addition, based on the operationalization by Mohr et al [53], eHealth-based self-management interventions included were further detailed: (1) intervention components (based on Morrison et al [54]; see Multimedia Appendix 3) - active intervention parts that support self-management behavior, including elements defined as what is provided to the user (eg, education materials, integrated alerts, and video conferencing options), how these elements are delivered (eg, plans and quizzes), and the subsequent intervention workflow defined as when they are delivered (eg, daily use) — and (2) intervention strategies - behavior change techniques [55] that underlie the intervention components (eg, role modeling if the Web-based education materials used include a video of patient who successfully manages his/her disease).

Outcome indicators were classified into 2 categories: effect outcome indicators and process outcome indicators [56]. Effect 
outcome indicators were outcomes related to self-management, health status, or cost-effectiveness, whereas process outcome indicators were outcomes on care process, health care delivery, or patient-health care interactions (eg, adherence and usability).

To allow for comparability, we classified the results reported as positive effect, no statistically significant effect, or mixed effect (see Textbox 2). No negative outcomes were reported in the studies included in this review. Only quantitative methods were used to measure effect outcome indicators, whereas mixed methods were used to measure some process outcome indicators. Hence, the classification of the results of the process outcome indicators slightly differs from that of the effect outcome indicators. Outcomes related to patients and care providers are reported separately.

The determinants of implementation of eHealth self-management interventions extracted were categorized following the widely cited framework by Fleuren et al [57]. This framework identifies 50 determinants of program implementation in 5 subgroups: (1) characteristics of the sociopolitical context, such as legislation; (2) characteristics of the organization, such as staff turnover; (3) characteristics of the person adopting the innovations (user of the innovation), such as knowledge; (4) characteristics of the innovation, such as complexity; and (5) innovation strategies, such as a training. For example, the study by McGillicuddy et al [36] included in our review mentioned that "six subjects did not complete the lead-in phase, 5 for technical reasons relating to poor internet at their home." This barrier was then mirrored to the 50 determinants in Fleuren framework and classified as a determinant related to the innovation and, more specifically, added to the determinant category perceived quality of eHealth intervention is excellent. In addition, in each subgroup, we identified the influence of the patients or care providers.

Textbox 2. Outcome indicators for electronic health self-management interventions.

- $\quad$ Effect outcome indicators

- Positive effect-if, after statistical analysis, a significant effect was reported

- No statistically significant effect-if, after statistical analysis, a nonstatistically significant effect was reported or if no statistical analysis was performed

- Mixed effect - if results that could be classified as both positive and no effect were reported

- $\quad$ Process outcome indicators

- Positive effect-if, after statistical analysis, a statistically significant effect was reported or if a positive effect or an improvement between certain points in time was reported (eg, interviews revealed that patients were highly satisfied with the electronic health application)

- No statistically significant effect-if, after statistical analysis, a nonsignificant effect was reported or if a no effect or no differences between certain points in time was reported

- Mixed effect-if results that could be classified as both positive and no effect were reported

\section{Results}

\section{Study Selection}

Our search retrieved 3307 articles in total. After removing 1497 duplicates, 1810 relevant articles were screened based on title and abstract. A total of 123 potentially relevant articles were screened full text. Of these papers, 2 described results of the same RCT [58,59] and were assessed jointly. Finally, 24 articles (comprising 23 studies) [33-36,58-77] were found eligible for inclusion in this review (Figure 1). 
Figure 1. Preferred Reporting Items for Systematic Reviews and Meta-Analyses flowchart of the systematic review. CKD: Chronic kidney disease; eHealth: electronic health.

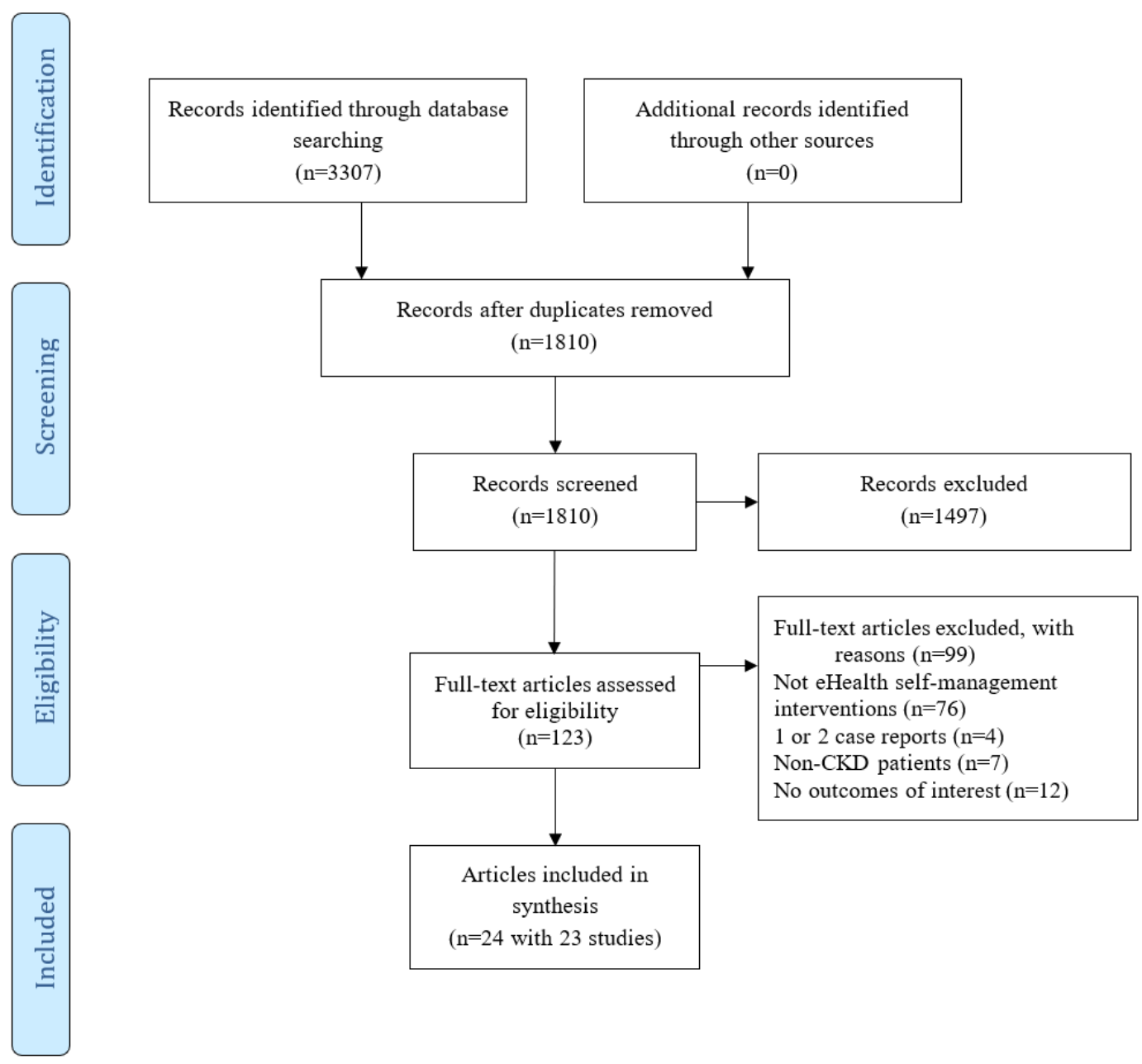

\section{Study Characteristics}

All 23 studies were published between 2005 and 2017, with 19 of them being conducted between 2012 and 2017 [33-36,58,64-77]. A total of 13 studies were conducted in the United States [33-36,58,60,62-65,69,71,72], followed by 2 studies in the United Kingdom [70,74]. The research designs used varied; the majority used an RCT design [33-36,58,63,64,66,70]. Most studies focused on the usability, acceptability, and feasibility of eHealth self-management interventions $\quad[36,58,61,64,65,67,69,71,72,74-77]$. Most participants are patients receiving hemodialysis [58,60,62-64,66,68,69,76,77]. Sample size at baseline ranged from 5 [67] to 601 [34]. Target population age ranged from 21 to 93 years. Recruitment mostly occurred via medical centers/hospitals [35,58,60-62,68,73]. Intervention duration ranged from 2 weeks [76] to 24 months [61]; 2 studies did not specify intervention duration [58,67]. A total of 10 studies performed a follow-up measurement [33,34,63,65,66,69-71,73,76]. Moreover, 12 studies included a control group, and 9 of those studies $[33,34,36,58,61,66,68,70,76]$ reported usual care or no internet-delivered intervention as control condition. The study characteristics have been presented in Multimedia Appendix 4.

\section{Quality Appraisal Scores of Studies}

Quality of the included articles varied (Table 1). A total of 3 articles [70,73,75] were awarded a 4-star rating, 11 $[33-36,59,63,65,66,69,74,76]$ a 3 -star rating, and 10 [58,60-62,64,67,68,71,72,77] a 2-star rating or lower. Articles with a 4-star rating scored higher on design, sampling, data collection, and ethics compared with those with a 3-star rating or lower. Moreover, 20 articles [34-36,59-70,72,73,75-77] provided insufficient details on their study design or rationale. Sampling method used (eg, randomly and purposively) was not reported in 10 articles $[35,60,62,65,67,71,73,74,76,77]$. Although both the number and characteristics of participants were described in most articles, 15 articles [58-62,64-69,71,74,76,77] did not specify the method of sample size calculation. A total of 10 articles [58-62,64,65,67,71,72] did not detail methods used to ensure the quality of the data collected or to reduce bias. On average, the lowest score was obtained on the ethics section. 
Table 1. Quality appraisal scores on the Crowe Critical Appraisal Tool.

\begin{tabular}{|c|c|c|c|c|c|c|c|c|c|c|}
\hline Study & $\begin{array}{l}\text { Total score } \\
(\text { maximum=40) }\end{array}$ & Star score ${ }^{\mathrm{a}}$ & Preamble & Introduction & Design & Sample & Data & Ethic & Result & Discussion \\
\hline $\begin{array}{l}\text { van Lint et al } \\
\text { (2015) [73] }\end{array}$ & 33 & 4-star & 4 & 4 & 4 & 4 & 4 & 4 & 4 & 5 \\
\hline $\begin{array}{l}\text { Blakeman et al } \\
\text { (2014) [70] }\end{array}$ & 32 & 4-star & 5 & 4 & 4 & 5 & 3 & 3 & 4 & 4 \\
\hline $\begin{array}{l}\text { Ong et al (2016) } \\
\text { [75] }\end{array}$ & 32 & 4-star & 4 & 4 & 4 & 4 & 4 & 4 & 4 & 4 \\
\hline $\begin{array}{l}\text { Forni Ogna et al } \\
\text { (2013) [66] }\end{array}$ & 31 & 3-star & 5 & 4 & 3 & 4 & 3 & 4 & 4 & 4 \\
\hline $\begin{array}{l}\text { Ishani et al (2016) } \\
\text { [34] }\end{array}$ & 31 & 3-star & 4 & 4 & 4 & 4 & 3 & 3 & 4 & 4 \\
\hline $\begin{array}{l}\text { Stark et al (2011) } \\
\text { [63] }\end{array}$ & 30 & 3-star & 4 & 4 & 4 & 4 & 3 & 3 & 4 & 4 \\
\hline $\begin{array}{l}\text { McGillicuddy et al } \\
\text { (2013) [36] }\end{array}$ & 30 & 3-star & 4 & 4 & 4 & 4 & 3 & 3 & 4 & 4 \\
\hline $\begin{array}{l}\text { Hayashi et al } \\
\text { (2017) [76] }\end{array}$ & 30 & 3-star & 5 & 4 & 3 & 3 & 4 & 3 & 4 & 4 \\
\hline $\begin{array}{l}\text { Diamantidis et al } \\
\text { (2013) [65] }\end{array}$ & 29 & 3-star & 5 & 4 & 4 & 3 & 3 & 4 & 3 & 3 \\
\hline $\begin{array}{l}\text { Reese et al (2017) } \\
\text { [35] }\end{array}$ & 29 & 3-star & 5 & 5 & 4 & 3 & 2 & 2 & 3 & 5 \\
\hline $\begin{array}{l}\text { Dey et al (2016) } \\
{[74]}\end{array}$ & 28 & 3-star & 3 & 4 & 3 & 3 & 3 & 4 & 4 & 4 \\
\hline $\begin{array}{l}\text { Berman et al } \\
\text { (2011) [59] }\end{array}$ & 27 & 3-star & 5 & 4 & 3 & 3 & 2 & 3 & 4 & 3 \\
\hline $\begin{array}{l}\text { Rifkin et al (2013) } \\
\text { [33] }\end{array}$ & 27 & 3-star & 5 & 4 & 3 & 4 & 3 & 2 & 3 & 3 \\
\hline $\begin{array}{l}\text { Welch et al (2013) } \\
\text { [69] }\end{array}$ & 27 & 3-star & 3 & 4 & 4 & 3 & 3 & 3 & 4 & 3 \\
\hline $\begin{array}{l}\text { Connelly et al } \\
\text { (2012) [64] }\end{array}$ & 26 & 2-star & 5 & 5 & 3 & 4 & 3 & 1 & 2 & 3 \\
\hline $\begin{array}{l}\text { Neumann et al } \\
\text { (2013) [68] }\end{array}$ & 26 & 2-star & 3 & 4 & 3 & 3 & 3 & 4 & 3 & 3 \\
\hline $\begin{array}{l}\text { Liu et al (2017) } \\
\text { [77] }\end{array}$ & 25 & 2-star & 4 & 5 & 3 & 2 & 3 & 1 & 3 & 4 \\
\hline $\begin{array}{l}\text { Diamantidis et al } \\
\text { (2015) [72] }\end{array}$ & 24 & 2-star & 3 & 4 & 3 & 4 & 1 & 3 & 3 & 3 \\
\hline $\begin{array}{l}\text { Minatodani et al } \\
\text { (2013) [58] }\end{array}$ & 23 & 2-star & 3 & 4 & 2 & 1 & 3 & 4 & 3 & 3 \\
\hline $\begin{array}{l}\text { Sevick et al (2005) } \\
\text { [60] }\end{array}$ & 22 & 2-star & 3 & 4 & 3 & 2 & 2 & 2 & 3 & 3 \\
\hline $\begin{array}{l}\text { Harrington et al } \\
\text { (2014) [71] }\end{array}$ & 20 & 1-star & 3 & 4 & 2 & 3 & 3 & 0 & 3 & 2 \\
\hline $\begin{array}{l}\text { Gallar et al (2007) } \\
\text { [61] }\end{array}$ & 18 & 1-star & 2 & 2 & 3 & 2 & 1 & 3 & 2 & 3 \\
\hline $\begin{array}{l}\text { Heiden et al (2013) } \\
\text { [67] }\end{array}$ & 18 & 1-star & 3 & 4 & 3 & 1 & 1 & 0 & 3 & 3 \\
\hline $\begin{array}{l}\text { Whitten et al } \\
\text { (2008) [62] }\end{array}$ & 14 & 1-star & 2 & 4 & 1 & 1 & 1 & 0 & 1 & 4 \\
\hline
\end{tabular}

${ }^{\mathrm{a}}$ 1-star: more than 1 SD below mean; 2-star, between 1 SD below mean and mean; 3-star, between mean and 1 SD above mean; 4-star, more than 1 SD above mean. 


\section{Description of Electronic Health Self-Management Interventions}

Major types of eHealth, functionalities, and key intervention components used are summarized in Tables 2 and 3. Most eHealth interventions evaluated included multiple components (multiple eHealth types) to improve patients' self-management (8/23 articles). Studies included did not provide detail on the specific intervention strategies underpinning these components, such as behavior change techniques. The most frequently used intervention component was self-monitoring (17/23 articles), followed by educational material or training (15/23 articles) and counseling (14/23 articles). Less frequently used intervention components were quizzes (3/23 articles) and interactive feedback from a device (4/23 articles). In addition, 5 studies reported that intervention development was guided by a specific theory.

Table 2. Descriptions of electronic health for each report included in the review.

\begin{tabular}{llll}
\hline Category of eHealth $^{\mathrm{a}}$ & Detailed eHealth & Functionality \\
\hline Personal digital assistant (references) & Dietary self-Monitoring: meals logs & Record \\
Sevick et al (2005) [60] & Dietary self-Monitoring: meals logs \\
Stark et al (2011) [63] & Dietary intake monitoring: self-monitor diet and feedback & Record \\
Connelly et al (2012) [64] & Electronic medication event monitoring: monitor adherence & Record \\
Forni Ogna et al (2013) [66] & Dietary intake monitoring: self-monitor diet and feedback & Record; communicate \\
Welch et al (2013) [69] & $\begin{array}{l}\text { Medication inquiry system: identifying the safety of medications with } \\
\text { impaired renal function }\end{array}$
\end{tabular}

Telemedicine (references)

Gallar et al (2007) [61]

Whitten et al (2008) [62]

\section{Computer (references)}

Harrington et al (2014) [71]

Ishani et al (2016) [34]

Heiden et al (2013) [67]

\section{Multiple components (references)}

McGillicuddy et al (2013) [36]

Minatodani et al (2013) [58], Self-monitoring devices

Berman et al (2011) [59]

Blakeman et al (2014) [70]

Dey et al (2016) [74]

Ong et al (2016) [75]

Hayashi et al (2017) [76]

Liu et al (2017) [77]

Wearable devices (references)

Neumann et al (2013) [68]

Rifkin et al (2013) [33]

van Lint et al (2015) [73]

Reese et al (2017) [35]

\footnotetext{
a eHealth: electronic health.
}

${ }^{\mathrm{b}} \mathrm{BP}$ : blood pressure. support to binder dosage

BP monitoring

Wireless pill bottle
Videoconferencing: connecting home to hospital

Videoconferencing: connecting clinics and health system

Tablet computer: recording data and reviewing medical findings

Touch screen computer with peripherals

Educational tool, food analyzer database and diet registration, and decision

Alert accessories linked to website/safe kidney care: offering information

$\mathrm{BP}^{\mathrm{b}}$ monitoring, electronic medication tray, and mobile phone

Website: tailoring access to community resources

Computer tablet, wearable devices, and Web portal

Smartphone, a Web-based dashboard application, and a data server

Self-management and recording system for dialysis (wearable devices, smartphone, and administrator module)

App installed on mobile, cloud server, and Web app

Telemetric weight monitoring

$\mathrm{BP}$ monitoring and creatine monitoring
Display; alert

Communicate

Communicate; education

Display; record; communicate; alert

Record; communicate

Communicate; education; record

Record; education

Alert; communicate

Record; communicate

Display; communicate

Record; alert

Record; alert; display

Record; alert; display

Record; alert; communicate

Record

Record

Record; alert 
Table 3. Descriptions of electronic health self-management interventions for each report included in the review.

\begin{tabular}{|c|c|c|c|c|c|c|c|c|c|c|c|}
\hline \multirow{2}{*}{$\begin{array}{l}\text { Category of } \\
\text { electronic } \\
\text { health }\end{array}$} & \multicolumn{10}{|c|}{ Intervention components } & \multirow{2}{*}{$\begin{array}{l}\text { Theory- } \\
\text { based }\end{array}$} \\
\hline & $\begin{array}{l}\text { Educa- } \\
\text { tional ma- } \\
\text { terial or } \\
\text { training }\end{array}$ & $\begin{array}{l}\text { Plan/ } \\
\text { goals }\end{array}$ & $\begin{array}{l}\text { Self- } \\
\text { monitor- } \\
\text { ing }\end{array}$ & $\begin{array}{l}\text { Interac- } \\
\text { tive feed- } \\
\text { back } \\
\text { from de- } \\
\text { vice }\end{array}$ & $\begin{array}{l}\text { Message/ } \\
\text { alert to } \\
\text { health } \\
\text { care- } \\
\text { givers }\end{array}$ & $\begin{array}{l}\text { Message/ } \\
\text { alerts to } \\
\text { patients } \\
\text { from de- } \\
\text { vice }\end{array}$ & $\begin{array}{l}\text { Message/ } \\
\text { alert to pa- } \\
\text { tients from } \\
\text { health care- } \\
\text { givers }\end{array}$ & Quizzes & Counseling & Daily use & \\
\hline
\end{tabular}

\section{Personal digital assistant (references)}

Sevick et al

(2005) [60]

Stark et al

(2011) [63]

Connelly et

al (2012)

[64]

Forni Ogna -

et al (2013)

[66]

Welch et al

(2013) [69]

Diaman-

tidis et al

(2015) [72]

Total

$5(83)$

$3(50) \quad 4(67)$

$2(33)$

0

$1(17)$

$2(33)$

0

$5(83)$

$2(33)$

$4(67)$

(\%)

\section{Telemedicine (references)}

\begin{tabular}{|c|c|c|c|c|c|c|}
\hline $\begin{array}{l}\text { Gallar et al } \\
\text { (2007) [61] }\end{array}$ & - & - & - & - & - & - \\
\hline $\begin{array}{l}\text { Whitten et } \\
\text { al (2008) }\end{array}$ & - & - & - & - & - & - \\
\hline
\end{tabular}

[62]

Total

$0(0)$

$0(0) \quad 0(0)$

$0(0)$

$0(0)$

$0(0)$

$0(0)$

$0(0)$

$2(100)$

$0(0)$

$0(0)$

(\%)

\section{Computer (references)}

Harrington
et al (2014)
[71]




\begin{tabular}{|c|c|c|c|c|c|c|c|c|c|c|c|}
\hline \multirow{2}{*}{$\begin{array}{l}\text { Category of } \\
\text { electronic } \\
\text { health }\end{array}$} & \multicolumn{10}{|c|}{ Intervention components } & \multirow{2}{*}{$\begin{array}{l}\text { Theory- } \\
\text { based }\end{array}$} \\
\hline & $\begin{array}{l}\text { Educa- } \\
\text { tional ma- } \\
\text { terial or } \\
\text { training }\end{array}$ & $\begin{array}{l}\text { Plan/ } \\
\text { goals }\end{array}$ & $\begin{array}{l}\text { Self- } \\
\text { monitor- } \\
\text { ing }\end{array}$ & $\begin{array}{l}\text { Interac- } \\
\text { tive feed- } \\
\text { back } \\
\text { from de- } \\
\text { vice }\end{array}$ & $\begin{array}{l}\text { Message/ } \\
\text { alert to } \\
\text { health } \\
\text { care- } \\
\text { givers }\end{array}$ & $\begin{array}{l}\text { Message/ } \\
\text { alerts to } \\
\text { patients } \\
\text { from de- } \\
\text { vice }\end{array}$ & $\begin{array}{l}\text { Message/ } \\
\text { alert to pa- } \\
\text { tients from } \\
\text { health care- } \\
\text { givers }\end{array}$ & Quizzes & Counseling & Daily use & \\
\hline $\begin{array}{l}\text { Minatodani } \\
\text { et al (2013) } \\
{[58] \text {, }} \\
\text { Berman et } \\
\text { al (2011) } \\
{[59]}\end{array}$ & $\checkmark$ & $\checkmark$ & $\checkmark$ & - & - & - & $\checkmark$ & $\checkmark$ & $\checkmark$ & - & - \\
\hline $\begin{array}{l}\text { Blakeman } \\
\text { et al (2014) } \\
{[70]}\end{array}$ & $\checkmark$ & - & - & - & - & - & - & - & $\checkmark$ & - & - \\
\hline $\begin{array}{l}\text { Dey et al } \\
\text { (2016) [74] }\end{array}$ & $\checkmark$ & - & $\checkmark$ & - & $\checkmark$ & - & $\checkmark$ & $\checkmark$ & $\checkmark$ & - & - \\
\hline $\begin{array}{l}\text { Ong et al } \\
\text { (2016) [75] }\end{array}$ & $\checkmark$ & $\checkmark$ & $\checkmark$ & $\checkmark$ & $\checkmark$ & $\checkmark$ & - & - & - & - & - \\
\hline $\begin{array}{l}\text { Hayashi et } \\
\text { al (2017) } \\
{[76]}\end{array}$ & - & $\checkmark$ & $\checkmark$ & - & - & $\checkmark$ & $\checkmark$ & - & $\checkmark$ & $\checkmark$ & - \\
\hline $\begin{array}{l}\text { Liu et al } \\
\text { (2017) [77] }\end{array}$ & - & - & $\checkmark$ & - & - & $\checkmark$ & - & $\checkmark$ & - & - & - \\
\hline $\begin{array}{l}\text { Total } \\
(\mathrm{N}=8), \mathrm{n} \\
(\%)\end{array}$ & $6(75)$ & $4(50)$ & $6(75)$ & $1(13)$ & $3(38)$ & $4(50)$ & $4(50)$ & $3(38)$ & $5(63)$ & $2(25)$ & $1(13)$ \\
\hline \multicolumn{12}{|c|}{ Wearable devices (references) } \\
\hline $\begin{array}{l}\text { Neumann } \\
\text { et al (2013) } \\
{[68]}\end{array}$ & - & $\checkmark$ & $\checkmark$ & - & $\checkmark$ & - & $\checkmark$ & - & $\checkmark$ & $\checkmark$ & - \\
\hline $\begin{array}{l}\text { Rifkin et al } \\
\text { (2013) [33] }\end{array}$ & $\checkmark$ & - & $\checkmark$ & - & - & - & $\checkmark$ & - & $\checkmark$ & - & - \\
\hline $\begin{array}{l}\text { van Lint et } \\
\text { al (2015) } \\
{[73]}\end{array}$ & $\checkmark$ & $\checkmark$ & $\checkmark$ & - & - & - & - & - & - & - & - \\
\hline $\begin{array}{l}\text { Reese et al } \\
\text { (2017) [35] }\end{array}$ & $\checkmark$ & $\checkmark$ & $\checkmark$ & $\checkmark$ & $\checkmark$ & $\checkmark$ & $\checkmark$ & - & - & - & - \\
\hline $\begin{array}{l}\text { Total } \\
(\mathrm{N}=4), \mathrm{n} \\
(\%)\end{array}$ & $3(75)$ & $3(75)$ & $4(100)$ & $1(25)$ & $2(50)$ & $1(25)$ & $3(75)$ & $0(0)$ & $2(50)$ & $1(25)$ & $0(0)$ \\
\hline
\end{tabular}

${ }^{\mathrm{a}}$ Not applicable.

\section{Summary of Results}

Tables 4 and 5 present the outcome indicators and the data collection tools used. Moreover, full details on the efficacy data reported in the included studies are included in Multimedia Appendix 5. Table 6 displays the determinants of implementation extracted. No articles reported any adverse outcomes of eHealth self-management interventions. 
Table 4. Summary of outcome indicators of electronic health self-management interventions.

\begin{tabular}{|c|c|c|c|c|}
\hline \multirow[t]{2}{*}{ Outcome category and indicator } & \multirow{2}{*}{$\begin{array}{l}\text { Total number of arti- } \\
\text { cles in each category }\end{array}$} & \multicolumn{3}{|l|}{ Effect and references } \\
\hline & & Positive, n (\%) & $\begin{array}{l}\text { No statistically significant effect, } \\
\mathrm{n}(\%)\end{array}$ & Mixed, n (\%) \\
\hline \multicolumn{5}{|l|}{ Patient effect outcome $(\mathrm{N}=33)$} \\
\hline Blood pressure & 5 & $4(80)[36,68,70,75]^{\mathrm{a}}$ & $1(20)[33]$ & $0(0)$ \\
\hline Quality of life & 4 & $1(25)[70]^{\mathrm{a}}$ & $2(50)[59,74]$ & $1(25)[76]$ \\
\hline Laboratory tests & 6 & $2(33)[66,68]^{\mathrm{a}}$ & $4(67)[60,62,75,76]$ & $0(0)$ \\
\hline Interdialytic weight gain & 4 & $1(25)[68]^{\mathrm{a}}$ & $3(75)[60,69,76]$ & $0(0)$ \\
\hline Morbidity and mortality & 2 & $0(0)$ & $2(100)[34,61]$ & $0(0)$ \\
\hline $\begin{array}{l}\text { Hospitalization rate and emer- } \\
\text { gency room visit }\end{array}$ & 3 & $2(67)[61,59]^{\mathrm{a}}$ & $1(33)[34]$ & $0(0)$ \\
\hline Medical cost & 2 & $1(50)[59]^{\mathrm{a}}$ & $1(50)[61]$ & $0(0)$ \\
\hline Cost-effectiveness & 1 & $1(100)[70]^{\mathrm{a}}$ & $0(0)$ & $0(0)$ \\
\hline Nutrition and dietary intake & 2 & $0(0)$ & $2(100)[62,69]$ & $0(0)$ \\
\hline Medication adherence & 4 & $3(75)[35,36,66]^{\mathrm{a}}$ & $1(25)[33]$ & $0(0)$ \\
\hline \multicolumn{5}{|l|}{ Process outcome $(\mathrm{N}=28)$} \\
\hline Acceptability & 6 & $6(100) ;[69,74,76]^{\mathrm{b}} ;[33,36,75]^{\mathrm{c}}$ & $0(0)$ & $0(0)$ \\
\hline Usability & 5 & $5(100) ;[64,67,76]^{\mathrm{b}} ;[62,77]^{\mathrm{c}}$ & $0(0)$ & $0(0)$ \\
\hline Satisfaction & 8 & $8(100) ;[36,58,71-74,76]^{\mathrm{b}} ;[75]^{\mathrm{c}}$ & $0(0)$ & $0(0)$ \\
\hline Adherence to intervention & 4 & $4(100) ;[35,63,73,75]^{\mathrm{b}}$ & $0(0)$ & $0(0)$ \\
\hline $\begin{array}{l}\text { First entry and length of dwell } \\
\text { time }\end{array}$ & 1 & $1(100) ;[65]^{b}$ & $0(0)$ & $0(0)$ \\
\hline Self-efficacy & 1 & $0(0)$ & $1(100) ;[69]^{\mathrm{b}}$ & $0(0)$ \\
\hline Perceived benefits & 1 & $0(0)$ & $1(100) ;[69]^{b}$ & $0(0)$ \\
\hline Perceived control & 1 & $1(100) ;[69]^{\mathrm{a}, \mathrm{b}}$ & $0(0)$ & $0(0)$ \\
\hline Recorded errors & 1 & $1(100) ;[72]^{b}$ & $0(0)$ & $0(0)$ \\
\hline
\end{tabular}

${ }^{\text {a }}$ Statistically significant.

${ }^{\mathrm{b}}$ Outcome related to patient.

${ }^{\mathrm{c}}$ Outcome related to both patient and care provider. 
Table 5. Summary of reported tools of outcome indicators.

\begin{tabular}{|c|c|}
\hline Outcome category and indicator & Reported data collection tools (number of articles) \\
\hline \multicolumn{2}{|l|}{ Patient effect outcome ( $N=33)$, all quantitative } \\
\hline Blood pressure & Readings (4) and dataset (1) \\
\hline Quality of life & $\begin{array}{l}\text { 36-item Short Form Health Survey (1), EuroQoL-5 Dimension (1), and 36-item Kidney } \\
\text { Disease Quality of Life survey (2) }\end{array}$ \\
\hline Laboratory tests & Medical records (6) \\
\hline Interdialytic weight gain & Medical records (4) \\
\hline Morbidity and mortality & Charlson comorbidity index (1) and records (1) \\
\hline Hospitalization rate and emergency room visit & Records (3) \\
\hline Medical cost & Records (2) \\
\hline Cost-effectiveness & Records (1) \\
\hline Nutrition and dietary intake & Clinical data (2) \\
\hline Medication adherence & $\begin{array}{l}\text { System data (2), adherence score calculation (1), and Morisky Medication Adherence } \\
\text { Scale (1) }\end{array}$ \\
\hline \multicolumn{2}{|l|}{ Process outcome $(\mathrm{N}=\mathbf{2 8})$} \\
\hline Acceptability & $\begin{array}{l}\text { Quantitative: questionnaires (1), recruitments and participation rate (1), QUEST }{ }^{\mathrm{a}} \text { and } \\
\text { retention rates (1), and average number of daily entries and completion rates (2); quanti- } \\
\text { tative and qualitative: number of assessments and semistructured interview (1) }\end{array}$ \\
\hline Usability & $\begin{array}{l}\text { Quantitative: survey (1) and questionnaire (2); qualitative: interview (1); quantitative and } \\
\text { qualitative: survey, interview, and system data (1) }\end{array}$ \\
\hline Satisfaction & $\begin{array}{l}\text { Quantitative: questionnaires and QUEST (5); qualitative: semistructured interview (2); } \\
\text { quantitative and qualitative: questionnaire and interview (1) }\end{array}$ \\
\hline Adherence to intervention & $\begin{array}{l}\text { Quantitative: system data (3) and Basel Assessment of Adherence to Immunosuppressive } \\
\text { Medications Scale (1) }\end{array}$ \\
\hline First entry and length of dwell time & Quantitative: frequency and number (1) \\
\hline Self-efficacy & Quantitative: cardiac diet self-efficacy and Fluid Self-Efficacy Scale (1) \\
\hline Perceived benefits & $\begin{array}{l}\text { Quantitative: Benefits of Sodium Adherence and a 9-item Benefits of Fluid Adherence } \\
\text { Scale (1) }\end{array}$ \\
\hline Perceived control & Quantitative: 7-item Mastery scale (1) \\
\hline Recorded errors & Quantitative: questionnaire and record (1) \\
\hline
\end{tabular}

${ }^{\mathrm{a}}$ QUEST: Quebec user evaluation of satisfaction with assistive technology. 
Table 6. Determinants of the implementation of electronic health self-management interventions for chronic kidney disease.

\begin{tabular}{lll}
\hline Determinants of interventions and details & References & \\
& If determinant is present & If determinant is exact opposite
\end{tabular}

\section{Sociopolitical context (patient)}

Awareness of potential health benefits of the eHealth ${ }^{\mathrm{a}}$ self-management intervention

Target population feels comfortable about eHealth use

\section{Organization (patient)}

Community resources (eg, activities, services, and applicable wireless fidelity connection at the users' location) available for implementation

\section{User}

\section{Patient}

Support from colleagues (eg, internet personnel)

Ability of health care professionals to monitor and, if necessary, anticipate on patient measurements online

Availability of sufficient skills/knowledge

eHealth technology is considered valuable by user

High self-efficacy

\section{Patient and care provider}

eHealth technology is considered valuable by user

Rifkin et al (2013) [33]

\section{Innovation}

Patient

Implementation of intervention is perceived as risk-free by user

Provision of warning/alert/reminder based on parameters monitored

Provision of real-time feedback (eg, amount of dietary intake, blood pressure value) based on patients' input

Stark et al (2011) [63]

McGillicuddy et al (2013) [36]

Rifkin et al (2013) [33], Reese et al (2017) [35], Berman et al (2011) [59], van Lint et al (2015) [73], Ong et al (2016) [75], Liu et al (2017) [77]

Diamantidis et al (2015) [72] [69]

McGillicuddy et al (2013) [36], Heiden et al (2013) [67], Blakeman et al (2014) [70] van Lint et al (2015) [73]

Harrington et al (2014) [71], Dey et al (2016) [74], Hayashi et al (2017) [76]

Reese et al (2017) [35], McGillicuddy et al (2013) [36], van Lint et al (2015) [73]

Sevick et al (2005) [60], Stark et al (2011) [63], Connelly et al (2012) [64], Hayashi et al (2017) [76]

Perceived quality of eHealth intervention is excellent

\section{Patient and care provider}

Interventions are compatible with existing work procedures

Implementation of intervention is perceived as advantageous by patient and care providers considering increasing access to health care services

High acceptability of eHealth

Rifkin et al (2013) [33]

Perceived quality of eHealth intervention is excel- Gallar et al (2007) [61] lent

\section{Innovation strategies (patient and care provider)}

Well planned/structured implementation process
Rifkin et al (2013) [33]

Whitten et al (2008) [62]

Liu et al (2017) [77]

\footnotetext{
${ }^{\mathrm{a}}$ eHealth: electronic health.

${ }^{\mathrm{b}}$ Not applicable.
}

Rifkin et al (2013) [33], McGillicuddy et al (2013) [36], Berman et al (2011) [59], Harrington et al (2014) [71] 


\section{Description of Effect Outcome Indicators}

The effect outcome indicators most frequently reported were laboratory tests (eg, serum albumin, C-reactive protein; 6/23 articles) and blood pressure (BP; 5/23 articles). Interdialytic weight gain (4/23 articles), QoL (4/23 articles), and medication adherence (4/23 articles) were also frequently reported. Finally, 2 studies assessed effects on morbidity and mortality, 2 evaluated changes in medical cost, and 1 performed a cost-effectiveness analysis.

Out of 5 studies, $4[36,68,70,75]$ reported a statistically significant positive effect on BP. Of the 2 studies $[59,61]$ that evaluated changes in medical costs, 1 [59] reported a significant reduction in costs in the intervention group. A study reported an incremental cost-effectiveness ratio of US $\$ 175$, showing that the implementation of a website-based self-management intervention for $\mathrm{CKD}$ patients was superior, considering effects and costs, to usual care [70]. Out of 3 studies, 2 [59,61] reported statistically significant improvements in hospitalization rates and emergency room visits. Out of 4 studies, 3 [35,36,66] reported statistically significant improvements in patients' medication adherence. Out of 4 studies, 1 [70] reported a statistically significant improvement on QoL.

\section{Description of Process Outcome Indicators}

The process outcome indicator satisfaction was reported in one-third of included studies. A total of 2 studies [58,75] used interviews to evaluate satisfaction in patients or care providers. Patients were reported to be satisfied with the use of at-home telehealth and appreciated its utility in managing their health [58]. Patients using a smartphone-based self-management system indicated feeling more confident and more in control of their condition; the nurses found that the system helped prioritize patients who needed more attention [75]. A total of 5 studies used questionnaires to evaluate satisfaction of patients $[36,71,72,74,76]$. These studies reported patients were highly satisfied with eHealth self-management interventions.

Acceptability was also frequently reported and mostly measured using questionnaires, retention rates, or system data $[33,36,69,74-76](6 / 23$ articles). All these studies reported that eHealth self-management interventions were acceptable to patients [33,36,69,74-76] and care providers [33,36]. Other process outcome indicators (including adherence to the intervention, first entry, length of dwell time, self-efficacy, perceived benefits, perceived control, and recorded errors) were less frequently used.

\section{Description of Implementation Determinants}

All but 4 studies $[34,65,66,68]$ reported on determinants of implementation. Studies included used various methods (eg, qualitative interview and quantitative data analysis) to evaluate determinants of implementation. The determinant ability of health care professionals to monitor and, if necessary, anticipate on patient measurements online is mostly reported to make patients feel safe while using eHealth interventions [77], thereby influencing patients' medication adherence [35] and adherence to interventions [35,73]. Moreover, availability of sufficient skills/knowledge [58,69,72] was reported as an important determinant to patients' use of the eHealth self-management interventions. In addition, the determinant provision of real-time feedback based on patients' input was frequently reported to influence patients' adherence to self-monitoring and healthy behaviors $[60,63,64,76]$. The determinant perceived quality of eHealth intervention is excellent [61] was cited to influence both patients' and care providers' use of the intervention. The percent agreement between the 2 reviewers' classification of the implementation determinants reported following the Fleuren framework was $76 \%$, which is considered acceptable [48]. Discrepancies in classification were discussed until consensus was reached.

\section{Discussion}

\section{Principal Findings}

The main findings and implications have been presented in Textbox 3 .

The evidence regarding the implementation and effectiveness of eHealth self-management interventions for CKD patients was reviewed. The 23 studies included were appraised on methodological quality, and all relevant data were extracted. Although the evidence base is still inconclusive, our review provides an indication that eHealth self-management interventions have the potential to improve CKD patients' management and health outcomes. Furthermore, high acceptability of and satisfaction with the eHealth interventions used were reported. Owing to the heterogeneity of the intervention components and outcomes measures used, we could not determine which intervention components contributed most to the effects found. The determinant ability of health care professionals to monitor and, if necessary, anticipate on patient measurements online was most frequently reported to influence implementation. The determinants reported were not quantified, and the relative importance of each determinant could not be determined.

Textbox 3. Main findings and implications for this study.

- Although the evidence base is still inconclusive, a majority of studies on electronic health (eHealth) self-management interventions report improvements on proximal outcomes (eg, blood pressure controlling) and mixed effects for more distal (eg, quality of life) outcomes.

- Evidence on the process level is more established; eHealth self-management interventions for chronic kidney disease patients are reported to be highly feasible, usable, and acceptable.

- To adequately assess eHealth intervention effect, future researchers should carefully consider their choice of outcomes (distal vs proximal) based on their sensitivity to capture meaningful change.

- Standardization of research design and methods in the evaluation of eHealth self-management interventions for chronic kidney disease patients is needed to optimize quality and comparability across studies and further elucidate which intervention components alone or in interaction contribute to the promising results found. 


\section{Comparison of Findings}

Most studies reported the evaluation of effect outcome indicators. The positive effects on patients' BP controlling $[36,68,70,75]$ and medication adherence $[35,36,66]$ were consistently reported; no adverse outcomes were reported. These findings correspond with another review on eHealth interventions in CKD [39]. Compared with standard outpatient-based management, eHealth self-management interventions have the potential to reduce health care delivery costs [78]. Although this potential reduction in costs is essential for policy makers and clinicians to adopt eHealth self-management interventions, health care expenditures were only assessed in 3 of the studies included, with only 1 performing a cost-effectiveness analysis [70]. Hence, we cannot yet determine if and how these interventions might reduce medical costs. This finding is consistent with similar reviews, which conclude that studies on the cost-effectiveness of eHealth self-management interventions are either conflicting or lacking [32,54]. As evidence on cost-effectiveness is important to support the potential scale-up of eHealth technology, further research is needed to broaden this evidence base. Regarding QoL, only 1 out of 4 studies reported a significant improvement. A possible explanation for this finding was the short follow-up period instated to capture changes in a distal outcome such as QoL [59]. As QoL in CKD is an independent predictor of mortality and hospitalization [79,80], and thus important to evaluate, we advise further research to assess QoL with a longer follow-up period.

In general, we found that eHealth self-management interventions were reported to be highly feasible, usable, and acceptable. However, we found great diversity in the use and operationalization of outcome indicators and how they were measured. For instance, a study reported acceptability by measuring adoption, adherence to the recommended intervention use, user satisfaction, and feature usage [75]. In contrast, other studies $[33,36]$ measured acceptability by asking patients "how acceptable they found the intervention" using a self-report scale. It is also notable that only 4 studies assessed implementation adherence, although finding no or limited intervention effects can be strongly related to patients' nonadherence to eHealth interventions as prescribed [81,82]. Examining implementation adherence can help resolve the black box of patients' adoption and continued use of the intervention, thereby preventing a type 3 error [83]. To tackle these issues, we advise researchers to use a standardized operationalization of process outcome indicators and measure implementation adherence to enable reliable interpretation of the intervention effect found.

Considering which outcomes are most sensitive to change is important. As eHealth interventions studies are mostly of short duration, they may not detect changes in distal outcomes (eg, QoL). Hence, effectivity might be easier to detect when proximal outcomes, close to the intervention strategies, are measured. For example, BP controlling can be an outcome sensitive to change if self-monitoring is the main intervention component. Functional outcomes (such as days needed to return to work), which can quantify patients' subjective perceptions of the effect of treatment on their daily life, might also be very sensitive to change by eHealth interventions [84,85]. Moreover, researchers should consider if their outcomes reflect meaningful change and provide a clear rationale for their choice of laboratory parameters. For example, using serum albumin as an indicator for dietary adherence might be of limited value as it is influenced by other CKD characteristics (eg, low dialysis dose) [60].

Furthermore, improving knowledge on the effect modifiers at play in eHealth self-management interventions for CKD patients is important. None of the included studies provided detail on potentially relevant effect modifiers. We can identify some possible modifying factors based on research focusing on self-management interventions in other chronic, noncommunicable diseases (NCDs). For instance, a longer intervention duration might positively modify the effect of self-management interventions [86]. In addition, the patients' health literacy level might modify intervention effect [87]. Self-management interventions for NCDs are mostly based on similar intervention principles and behavior change techniques. Moreover, the characteristics of patients suffering from NCDs are often similar. We, therefore, argue that the modifiers found to influence the outcomes of self-management interventions for NCDs in general might also be applicable for similar interventions targeting CKD patients. However, more research is needed to identify effect modifiers to self-management interventions targeting CKD and explore possible strategies to impact these factors.

\section{Electronic Health Self-Management Interventions}

A large variety of eHealth self-management intervention components were used in the included studies (eg, self-monitoring and education), and the results differed greatly. These findings make it difficult and possibly premature to formulate a potentially ideal palette of eHealth self-management intervention components for CKD patients. However, reviewing results make it possible to identify which intervention components might be more promising than others. For instance, self-monitoring and the use of messages or alerts to nudge patient toward displaying healthy behaviors (see Multimedia Appendix 6) were most commonly reported as the effective components to optimize patient self-management skills.

Furthermore, few of the interventions studied were theory-based. The authors recommend that a strong theoretical foundation is necessary for the planning, design, evaluation, and implementation of eHealth self-management interventions [88]. We recommend building eHealth self-management interventions based on established behavior change techniques, such as formulated in the Behavior Change Techniques taxonomy [55]. Moreover, the use of cocreation methods and appreciative inquiry (such as described in the Center for eHealth Research and Disease Management [89] roadmap for eHealth development) can improve intervention fit with the needs and priorities expressed by professionals and patients.

\section{Determinants of Implementation}

Ability of health care professionals to monitor and, if necessary, anticipate patient measurements online was reported as an important determinant of implementation. We argue that this ability of professionals to anticipate and act upon patient measurements might reduce patients' feeling of isolation and/or 
anxiety caused by independently conducted treatments at home [77] and thereby increase patients' adherence to implementation. In addition, availability of sufficient skills/knowledge was important for users to continue their use of eHealth technology. If participants are unfamiliar with the use of eHealth, this has been reported to limit their acceptance of eHealth interventions $[58,69]$. Proper training and tailored tutorials are needed to guide eHealth implementation to optimize knowledge and skills and promote intervention uptake $[67,72]$. The included studies used various methods to evaluate determinants of implementation. We suggest that future research should use validated tools for measuring implementation quality and related determinants, such as the Measurement Instrument for Determinants of Innovations questionnaire and Determinants of Implementation Behavior Questionnaire [90,91].

\section{Study Quality and Characteristics}

Most studies were appraised to be of low to moderate quality. There is a heterogeneity of outcome measurement tools and reporting styles used in the articles included in this review. Therefore, we advise researchers to develop a more standardized approach to the use of outcome measures, guided by, for instance, the formulation of an International Consortium for Health Outcomes Measurement standard set for CKD [92]. In addition, we argue that detailed description and a thorough analysis of study design, methods, and intervention components used, based on a published theoretical framework such as Consolidated Standards of Reporting Trials-eHealth [93], can improve reporting and provide a basis for evaluating the validity and applicability of eHealth trials.

Data on eHealth self-management interventions for CKD patients in developing countries are still lacking, which corresponds with other reviews on eHealth interventions [94,95]. The need to perform such research in developing countries is high. eHealth interventions in these countries have the potential to improve the accessibility and cost-effectiveness of local care and ensure timely delivery of care to rural areas and diverse populations [20,24,96]. Furthermore, 9 studies had an intervention duration of fewer than 6 months. Few studies conducted a follow-up measurement. Forni Ogna et al [66] reported that the positive intervention effects were maintained only during the monitoring period; these effects had vanished 3 months after interruption of the drug adherence monitoring. This finding underlines that the effectiveness of eHealth self-management interventions should be tested during a longer study period and with follow-up measurements.

Of note, 3 studies with fewer than 10 participants were included. One might argue that such studies do not provide robust, generalizable evidence and should be excluded based only on their sample size. However, high-level evidence on the effectiveness of eHealth self-management interventions for CKD patients, for instance, generated by large RCTs, is very limited. Hence, studies with less robust designs are included, as in this stage, we feel that all evidence should be accumulated and taken into account as to broaden our view and deepen our understanding of the usability, implementability, and effectiveness of eHealth self-management interventions for CKD patients. Moreover, this decision is supported by similar systematic reviews on the effectivity of eHealth interventions that also included studies with smaller sample sizes $[95,97,98]$. That being said, results of this review should be interpreted with some caution.

\section{Strengths and Limitations}

To our knowledge, this is the first systematic review to evaluate the entire spectrum of studies focusing on eHealth self-management interventions for CKD patients. Our review has some strengths. First, PRISMA guidelines were followed, and a robust search strategy was used in 8 databases. Second, a comprehensive analysis was conducted on the intervention components, outcome indicators, and determinants from the various studies. The kappa value and percent agreement obtained, and thus inter-rater reliability, showed that the validity of the appraisal could be considered fair. Finally, any discrepancies were discussed until consensus was reached.

Nevertheless, several limitations need to be addressed. First, as articles only published in English were included, some relevant articles might have been missed. Second, substantial heterogeneity of interventions and outcome measures made it difficult to draw firm conclusions about the evidence emerging from these studies, and results should be interpreted with caution.

\section{Conclusions}

This review provides a comprehensive overview of studies evaluating eHealth self-management interventions for CKD patients. eHealth self-management interventions show promise to improve health outcomes in CKD patients. To adequately assess eHealth intervention effect, future researchers should carefully consider their choice of outcomes (distal vs proximal) based on their sensitivity to capture meaningful change. Also, to enable the standard design and scale-up of effective eHealth self-management interventions for CKD patients, a more detailed understanding of which individual intervention components lead to health outcome improvement and which determinants of the implementation can promote adherence and satisfaction with care is needed.

\section{Acknowledgments}

This study was funded by the China Scholarship Council.

\section{Conflicts of Interest}

None declared. 


\section{Multimedia Appendix 1}

Search strategy.

[PDF File (Adobe PDF File), 222 KB-Multimedia Appendix 1]

\section{Multimedia Appendix 2}

Major types of electronic health.

[PDF File (Adobe PDF File), 193 KB-Multimedia Appendix 2]

\section{Multimedia Appendix 3}

Electronic health self-management intervention components.

[PDF File (Adobe PDF File), 283 KB-Multimedia Appendix 3]

\section{Multimedia Appendix 4}

Study characteristics.

[PDF File (Adobe PDF File), 170 KB-Multimedia Appendix 4]

\section{Multimedia Appendix 5}

Effects and references of outcome indicators.

[PDF File (Adobe PDF File), 3768 KB-Multimedia Appendix 5]

\section{Multimedia Appendix 6}

Most frequently recommended electronic health self-management intervention components.

[PDF File (Adobe PDF File), 193 KB-Multimedia Appendix 6]

\section{References}

1. Hill NR, Fatoba ST, Oke JL, Hirst JA, O'Callaghan CA, Lasserson DS, et al. Global prevalence of chronic kidney disease - a systematic review and meta-analysis. PLoS One 2016;11(7):e0158765 [FREE Full text] [doi: 10.1371/journal.pone.0158765] [Medline: 27383068]

2. Zhang L, Wang F, Wang L, Wang W, Liu B, Liu J, et al. Prevalence of chronic kidney disease in China: a cross-sectional survey. Lancet 2012 Mar 3;379(9818):815-822. [doi: 10.1016/S0140-6736(12)60033-6] [Medline: 22386035]

3. GBD 2013 Mortality and Causes of Death Collaborators. Global, regional, and national age-sex specific all-cause and cause-specific mortality for 240 causes of death, 1990-2013: a systematic analysis for the global burden of disease study 2013. Lancet 2015 Jan 10;385(9963):117-171 [FREE Full text] [doi: 10.1016/S0140-6736(14)61682-2] [Medline: 25530442]

4. Zhang Q, Rothenbacher D. Prevalence of chronic kidney disease in population-based studies: systematic review. BMC Public Health 2008 Apr 11;8:117 [FREE Full text] [doi: 10.1186/1471-2458-8-117] [Medline: 18405348]

5. National Kidney Foundation. K/DOQI clinical practice guidelines for chronic kidney disease: evaluation, classification, and stratification. Am J Kidney Dis 2002 Feb;39(2 Suppl 1):S1-266. [Medline: 11904577]

6. Couser WG, Remuzzi G, Mendis S, Tonelli M. The contribution of chronic kidney disease to the global burden of major noncommunicable diseases. Kidney Int 2011 Dec;80(12):1258-1270 [FREE Full text] [doi: 10.1038/ki.2011.368] [Medline: 21993585]

7. Gansevoort RT, Correa-Rotter R, Hemmelgarn BR, Jafar TH, Heerspink HJL, Mann JF, et al. Chronic kidney disease and cardiovascular risk: epidemiology, mechanisms, and prevention. Lancet 2013 Jul 27;382(9889):339-352. [doi: 10.1016/S0140-6736(13)60595-4] [Medline: 23727170]

8. Braun L, Sood V, Hogue S, Lieberman B, Copley-Merriman C. High burden and unmet patient needs in chronic kidney disease. Int J Nephrol Renovasc Dis 2012;5:151-163 [FREE Full text] [doi: 10.2147/IJNRD.S37766] [Medline: 23293534]

9. Chin HJ, Song YR, Lee JJ, Lee SB, Kim KW, Na KY, et al. Moderately decreased renal function negatively affects the health-related quality of life among the elderly Korean population: a population-based study. Nephrol Dial Transplant 2008 Sep;23(9):2810-2817. [doi: 10.1093/ndt/gfn132] [Medline: 18372390$]$

10. Etgen T, Chonchol M, Förstl H, Sander D. Chronic kidney disease and cognitive impairment: a systematic review and meta-analysis. Am J Nephrol 2012;35(5):474-482 [FREE Full text] [doi: 10.1159/000338135] [Medline: 22555151]

11. Lorig KR, Holman HR. Self-management education: history, definition, outcomes, and mechanisms. Ann Behav Med 2003 Aug;26(1):1-7. [doi: 10.1207/S15324796ABM2601 01] [Medline: 12867348]

12. Bonner A, Havas K, Douglas C, Thepha T, Bennett P, Clark R. Self-management programmes in stages 1-4 chronic kidney disease: a literature review. J Ren Care 2014 Sep;40(3):194-204. [doi: 10.1111/jorc.12058] [Medline: 24628848] 
13. Moattari M, Ebrahimi M, Sharifi N, Rouzbeh J. The effect of empowerment on the self-efficacy, quality of life and clinical and laboratory indicators of patients treated with hemodialysis: a randomized controlled trial. Health Qual Life Outcomes 2012 Sep 20;10:115 [FREE Full text] [doi: 10.1186/1477-7525-10-115] [Medline: 22992449]

14. Boots LM, de Vugt ME, Kempen GI, Verhey FR. Effectiveness of a blended care self-management program for caregivers of people with early-stage dementia (Partner In Balance): randomized controlled trial. J Med Internet Res 2018 Jul 13;20(7):e10017 [FREE Full text] [doi: 10.2196/10017] [Medline: 30006327]

15. Karamanidou C, Clatworthy J, Weinman J, Horne R. A systematic review of the prevalence and determinants of nonadherence to phosphate binding medication in patients with end-stage renal disease. BMC Nephrol 2008 Jan 31;9:2 [FREE Full text] [doi: 10.1186/1471-2369-9-2] [Medline: 18237373]

16. Ghimire S, Castelino RL, Lioufas NM, Peterson GM, Zaidi ST. Nonadherence to medication therapy in haemodialysis patients: a systematic review. PLoS One 2015;10(12):e0144119 [FREE Full text] [doi: 10.1371/journal.pone.0144119] [Medline: 26636968]

17. Donald M, Kahlon BK, Beanlands H, Straus S, Ronksley P, Herrington G, et al. Self-management interventions for adults with chronic kidney disease: a scoping review. BMJ Open 2018 Mar 22;8(3):e019814 [FREE Full text] [doi: 10.1136/bmjopen-2017-019814] [Medline: 29567848]

18. Havas K, Douglas C, Bonner A. Closing the loop in person-centered care: patient experiences of a chronic kidney disease self-management intervention. Patient Prefer Adherence 2017;11:1963-1973 [FREE Full text] [doi: 10.2147/PPA.S147831] [Medline: 29238172]

19. Slater H, Dear BF, Merolli MA, Li LC, Briggs AM. Use of ehealth technologies to enable the implementation of musculoskeletal models of care: evidence and practice. Best Pract Res Clin Rheumatol 2016 Jun;30(3):483-502 [FREE Full text] [doi: 10.1016/j.berh.2016.08.006] [Medline: 27886943]

20. Schwamm LH, Chumbler N, Brown E, Fonarow GC, Berube D, Nystrom K, American Heart Association Advocacy Coordinating Committee. Recommendations for the implementation of telehealth in cardiovascular and stroke care: a policy statement from the American Heart Association. Circulation 2017 Feb 14;135(7):e24-e44. [doi: 10.1161/CIR.0000000000000475] [Medline: 27998940]

21. Larsen-Cooper E, Bancroft E, Rajagopal S, O'Toole M, Levin A. Scale matters: a cost-outcome analysis of an m-health intervention in Malawi. Telemed J E Health 2016 Apr;22(4):317-324 [FREE Full text] [doi: 10.1089/tmj.2015.0060] [Medline: 26348994]

22. Eysenbach G. What is e-health? J Med Internet Res 2001;3(2):E20 [FREE Full text] [doi: 10.2196/jmir.3.2.e20] [Medline: 11720962]

23. Slev VN, Pasman HR, Eeltink CM, van Uden-Kraan CF, Verdonck-de Leeuw IM, Francke AL. Self-management support and ehealth for patients and informal caregivers confronted with advanced cancer: an online focus group study among nurses. BMC Palliat Care 2017 Nov 21;16(1):55 [FREE Full text] [doi: 10.1186/s12904-017-0238-4] [Medline: 29162081]

24. Black AD, Car J, Pagliari C, Anandan C, Cresswell K, Bokun T, et al. The impact of ehealth on the quality and safety of health care: a systematic overview. PLoS Med 2011 Jan 18;8(1):e1000387 [FREE Full text] [doi:

10.1371/journal.pmed.1000387] [Medline: 21267058]

25. Bertz F, Winkvist A, Brekke HK. Sustainable weight loss among overweight and obese lactating women is achieved with an energy-reduced diet in line with dietary recommendations: results from the LEVA randomized controlled trial. J Acad Nutr Diet 2015 Jan;115(1):78-86. [doi: 10.1016/j.jand.2014.05.017] [Medline: 25088520]

26. Herring SJ, Cruice JF, Bennett GG, Rose MZ, Davey A, Foster GD. Preventing excessive gestational weight gain among African American women: a randomized clinical trial. Obesity (Silver Spring) 2016 Jan;24(1):30-36 [FREE Full text] [doi: 10.1002/oby.21240] [Medline: 26592857]

27. Taylor GM, Dalili MN, Semwal M, Civljak M, Sheikh A, Car J. Internet-based interventions for smoking cessation. Cochrane Database Syst Rev 2017 Sep 4;9:CD007078 [FREE Full text] [doi: 10.1002/14651858.CD007078.pub5] [Medline: 28869775]

28. Clarke G, Eubanks D, Reid E, Kelleher C, O'Connor E, DeBar LL, et al. Overcoming depression on the internet (ODIN) (2): a randomized trial of a self-help depression skills program with reminders. J Med Internet Res 2005 Jun;7(2):e16 [FREE Full text] [doi: 10.2196/jmir.7.2.e16] [Medline: 15998607]

29. Steventon A, Bardsley M, Billings J, Dixon J, Doll H, Hirani S, Whole System Demonstrator Evaluation Team. Effect of telehealth on use of secondary care and mortality: findings from the Whole System Demonstrator cluster randomised trial. Br Med J 2012 Jun 21;344:e3874 [FREE Full text] [doi: 10.1136/bmj.e3874] [Medline: 22723612]

30. Seiler A, Klaas V, Tröster G, Fagundes CP. eHealth and mHealth interventions in the treatment of fatigued cancer survivors: a systematic review and meta-analysis. Psychooncology 2017 Sep;26(9):1239-1253. [doi: 10.1002/pon.4489] [Medline: 28665554]

31. Palacios J, Lee GA, Duaso M, Clifton A, Norman IJ, Richards D, et al. Internet-delivered self-management support for improving coronary heart disease and self-management-related outcomes: a systematic review. J Cardiovasc Nurs 2017;32(4):E9-23 [FREE Full text] [doi: 10.1097/JCN.0000000000000392] [Medline: 28107251]

32. Jackson BD, Gray K, Knowles SR, de Cruz P. Ehealth technologies in inflammatory bowel disease: a systematic review. J Crohns Colitis 2016 Sep;10(9):1103-1121. [doi: 10.1093/ecco-jcc/jjw059] [Medline: 26928960] 
33. Rifkin DE, Abdelmalek JA, Miracle CM, Low C, Barsotti R, Rios P, et al. Linking clinic and home: a randomized, controlled clinical effectiveness trial of real-time, wireless blood pressure monitoring for older patients with kidney disease and hypertension. Blood Press Monit 2013 Feb;18(1):8-15 [FREE Full text] [doi: 10.1097/MBP.0b013e32835d126c] [Medline: 23275313]

34. Ishani A, Christopher J, Palmer D, Otterness S, Clothier B, Nugent S, Center for Innovative Kidney Care. Telehealth by an interprofessional team in patients with CKD: a randomized controlled trial. Am J Kidney Dis 2016 Jul;68(1):41-49. [doi: 10.1053/j.ajkd.2016.01.018] [Medline: 26947216]

35. Reese PP, Bloom RD, Trofe-Clark J, Mussell A, Leidy D, Levsky S, et al. Automated reminders and physician notification to promote immunosuppression adherence among kidney transplant recipients: a randomized trial. Am J Kidney Dis 2017 Mar;69(3):400-409. [doi: 10.1053/j.ajkd.2016.10.017] [Medline: 27940063]

36. McGillicuddy JW, Gregoski MJ, Weiland AK, Rock RA, Brunner-Jackson BM, Patel SK, et al. Mobile health medication adherence and blood pressure control in renal transplant recipients: a proof-of-concept randomized controlled trial. JMIR Res Protoc 2013 Sep 4;2(2):e32 [FREE Full text] [doi: 10.2196/resprot.2633] [Medline: 24004517]

37. Campbell J, Porter J. Dietary mobile apps and their effect on nutritional indicators in chronic renal disease: a systematic review. Nephrology (Carlton) 2015 Oct;20(10):744-751. [doi: 10.1111/nep.12500] [Medline: 25959301]

38. He T, Liu X, Li Y, Wu Q, Liu M, Yuan H. Remote home management for chronic kidney disease: a systematic review. J Telemed Telecare 2017 Jan;23(1):3-13. [doi: 10.1177/1357633X15626855] [Medline: 27269795]

39. Jeddi FR, Nabovati E, Amirazodi S. Features and effects of information technology-based interventions to improve self-management in chronic kidney disease patients: a systematic review of the literature. J Med Syst 2017 Sep 18;41(11):170. [doi: 10.1007/s10916-017-0820-6] [Medline: 28920174]

40. Moher D, Liberati A, Tetzlaff J, Altman DG, PRISMA Group. Preferred reporting items for systematic reviews and meta-analyses: the PRISMA statement. PLoS Med 2009 Jul 21;6(7):e1000097 [FREE Full text] [doi:

10.1371/journal.pmed.1000097] [Medline: 19621072]

41. Kaltenthaler E, Sutcliffe P, Parry G, Beverley C, Rees A, Ferriter M. The acceptability to patients of computerized cognitive behaviour therapy for depression: a systematic review. Psychol Med 2008 Nov;38(11):1521-1530. [doi: 10.1017/S0033291707002607] [Medline: 18205964]

42. Crowe M, Sheppard L. A general critical appraisal tool: an evaluation of construct validity. Int J Nurs Stud 2011 Dec;48(12):1505-1516. [doi: 10.1016/j.ijnurstu.2011.06.004] [Medline: 21802686]

43. Crowe M, Sheppard L, Campbell A. Comparison of the effects of using the Crowe Critical Appraisal Tool versus informal appraisal in assessing health research: a randomised trial. Int J Evid Based Healthc 2011 Dec;9(4):444-449. [doi: 10.1111/j.1744-1609.2011.00237.x] [Medline: 22093394]

44. Crowe M, Sheppard L, Campbell A. Reliability analysis for a proposed critical appraisal tool demonstrated value for diverse research designs. J Clin Epidemiol 2012 Apr;65(4):375-383. [doi: 10.1016/j.jclinepi.2011.08.006] [Medline: 22078576]

45. Corrigan FM, Broome H, Dorris L. A systematic review of psychosocial interventions for children and young people with epilepsy. Epilepsy Behav 2016 Mar;56:99-112. [doi: 10.1016/j.yebeh.2016.01.005] [Medline: 26859319]

46. Crowe L, Chang A, Fraser JA, Gaskill D, Nash R, Wallace K. Systematic review of the effectiveness of nursing interventions in reducing or relieving post-operative pain. Int J Evid Based Healthc 2008 Dec;6(4):396-430. [doi: 10.1111/j.1744-1609.2008.00113.x] [Medline: 21631835]

47. van der Kleij R, Coster N, Verbiest M, van Assema P, Paulussen T, Reis R, et al. Implementation of intersectoral community approaches targeting childhood obesity: a systematic review. Obes Rev 2015 Jun;16(6):454-472. [doi: 10.1111/obr.12273] [Medline: 25824957]

48. Fleiss JL. Statistical Methods for Rates and Proportions. Second Edition. New York: Wiley-Interscience; 1981.

49. Lipsey MW, Wilson DB. Practical Meta-Analysis. First Edition. Thousand Oaks, CA: Sage Publications; 2001.

50. Vegesna A, Tran M, Angelaccio M, Arcona S. Remote patient monitoring via non-invasive digital technologies: a systematic review. Telemed J E Health 2017 Jan;23(1):3-17 [FREE Full text] [doi: 10.1089/tmj.2016.0051] [Medline: 27116181]

51. Powell AC, Landman AB, Bates DW. In search of a few good apps. J Am Med Assoc 2014 May 14;311(18):1851-1852. [doi: 10.1001/jama.2014.2564] [Medline: 24664278]

52. Chaet AV, Morshedi B, Wells KJ, Barnes LE, Valdez R. Spanish-language consumer health information technology interventions: a systematic review. J Med Internet Res 2016 Aug 10;18(8):e214 [FREE Full text] [doi: 10.2196/jmir.5794] [Medline: 27511437]

53. Mohr DC, Schueller SM, Riley WT, Brown CH, Cuijpers P, Duan N, et al. Trials of intervention principles: evaluation methods for evolving behavioral intervention technologies. J Med Internet Res 2015 Jul 8;17(7):e166 [FREE Full text] [doi: 10.2196/jmir.4391] [Medline: 26155878]

54. Morrison D, Wyke S, Agur K, Cameron EJ, Docking RI, Mackenzie AM, et al. Digital asthma self-management interventions: a systematic review. J Med Internet Res 2014 Feb 18;16(2):e51 [FREE Full text] [doi: 10.2196/jmir.2814] [Medline: 24550161]

55. Michie S, Richardson M, Johnston M, Abraham C, Francis J, Hardeman W, et al. The behavior change technique taxonomy (v1) of 93 hierarchically clustered techniques: building an international consensus for the reporting of behavior change interventions. Ann Behav Med 2013 Aug;46(1):81-95. [doi: 10.1007/s12160-013-9486-6] [Medline: 23512568] 
56. Beratarrechea A, Lee AG, Willner JM, Jahangir E, Ciapponi A, Rubinstein A. The impact of mobile health interventions on chronic disease outcomes in developing countries: a systematic review. Telemed J E Health 2014 Jan;20(1):75-82 [FREE Full text] [doi: 10.1089/tmj.2012.0328] [Medline: 24205809]

57. Fleuren M, Wiefferink K, Paulussen T. Determinants of innovation within health care organizations: literature review and Delphi study. Int J Qual Health Care 2004 Apr;16(2):107-123. [doi: 10.1093/intqhc/mzh030] [Medline: 15051705]

58. Minatodani DE, Chao PJ, Berman SJ. Home telehealth: facilitators, barriers, and impact of nurse support among high-risk dialysis patients. Telemed J E Health 2013 Aug;19(8):573-578. [doi: 10.1089/tmj.2012.0201] [Medline: 23742629]

59. Berman SJ, Wada C, Minatodani D, Halliday T, Miyamoto R, Lindo J, et al. Home-based preventative care in high-risk dialysis patients: a pilot study. Telemed J E Health 2011 May;17(4):283-287. [doi: 10.1089/tmj.2010.0169] [Medline: 21480788]

60. Sevick MA, Piraino B, Sereika S, Starrett T, Bender C, Bernardini J, et al. A preliminary study of PDA-based dietary self-monitoring in hemodialysis patients. J Ren Nutr 2005 Jul;15(3):304-311. [doi: 10.1016/j.jrn.2005.04.003] [Medline: 16007560]

61. Gallar P, Vigil A, Rodriguez I, Ortega O, Gutierrez M, Hurtado J, et al. Two-year experience with telemedicine in the follow-up of patients in home peritoneal dialysis. J Telemed Telecare 2007;13(6):288-292. [doi: 10.1258/135763307781644906] [Medline: 17785025]

62. Whitten P, Buis L. Use of telemedicine for haemodialysis: perceptions of patients and health-care providers, and clinical effects. J Telemed Telecare 2008;14(2):75-78. [doi: 10.1258/jtt.2007.070411] [Medline: 18348752]

63. Stark S, Snetselaar L, Piraino B, Stone RA, Kim S, Hall B, et al. Personal digital assistant-based self-monitoring adherence rates in 2 dialysis dietary intervention pilot studies: BalanceWise-HD and BalanceWise-PD. J Ren Nutr 2011 Nov;21(6):492-498 [FREE Full text] [doi: 10.1053/j.jrn.2010.10.026] [Medline: 21420316]

64. Connelly K, Siek KA, Chaudry B, Jones J, Astroth K, Welch JL. An offline mobile nutrition monitoring intervention for varying-literacy patients receiving hemodialysis: a pilot study examining usage and usability. J Am Med Inform Assoc 2012;19(5):705-712 [FREE Full text] [doi: 10.1136/amiajnl-2011-000732] [Medline: 22582206]

65. Diamantidis CJ, Fink W, Yang S, Zuckerman MR, Ginsberg J, Hu P, et al. Directed use of the internet for health information by patients with chronic kidney disease: prospective cohort study. J Med Internet Res 2013 Nov 15;15(11):e251 [FREE Full text] [doi: 10.2196/jmir.2848] [Medline: 24240617]

66. Ogna VF, Pruijm M, Zweiacker C, Wuerzner G, Tousset E, Burnier M. Clinical benefits of an adherence monitoring program in the management of secondary hyperparathyroidism with cinacalcet: results of a prospective randomized controlled study. Biomed Res Int 2013;2013:104892 [FREE Full text] [doi: 10.1155/2013/104892] [Medline: 23971019]

67. Heiden S, Buus AA, Jensen MH, Hejlesen OK. A diet management information and communication system to help chronic kidney patients cope with diet restrictions. Stud Health Technol Inform 2013;192:543-547. [doi: 10.3233/978-1-61499-289-9-543] [Medline: 23920614]

68. Neumann CL, Wagner F, Menne J, Brockes C, Schmidt-Weitmann S, Rieken EM, et al. Body weight telemetry is useful to reduce interdialytic weight gain in patients with end-stage renal failure on hemodialysis. Telemed J E Health 2013 Jun;19(6):480-486. [doi: 10.1089/tmj.2012.0188] [Medline: 23614336]

69. Welch JL, Astroth KS, Perkins SM, Johnson CS, Connelly K, Siek KA, et al. Using a mobile application to self-monitor diet and fluid intake among adults receiving hemodialysis. Res Nurs Health 2013 Jun;36(3):284-298 [FREE Full text] [doi: 10.1002/nur.21539] [Medline: 23512869]

70. Blakeman T, Blickem C, Kennedy A, Reeves D, Bower P, Gaffney H, et al. Effect of information and telephone-guided access to community support for people with chronic kidney disease: randomised controlled trial. PLoS One 2014;9(10):e109135 [FREE Full text] [doi: 10.1371/journal.pone.0109135] [Medline: 25330169]

71. Harrington DM, Myers L, Eisenman K, Bhise V, Nayak KS, Rosner MH. The use of a tablet computer platform to optimize the care of patients receiving peritoneal dialysis: a pilot study. Blood Purif 2014;37(4):311-315. [doi: 10.1159/000365440] [Medline: 25170838]

72. Diamantidis CJ, Ginsberg JS, Yoffe M, Lucas L, Prakash D, Aggarwal S, et al. Remote usability testing and satisfaction with a mobile health medication inquiry system in CKD. Clin J Am Soc Nephrol 2015 Aug 7;10(8):1364-1370 [FREE Full text] [doi: 10.2215/CJN.12591214] [Medline: 26220816]

73. van Lint CL, van der Boog PJ, Wang W, Brinkman WP, Rövekamp TJ, Neerincx MA, et al. Patient experiences with self-monitoring renal function after renal transplantation: results from a single-center prospective pilot study. Patient Prefer Adherence 2015;9:1721-1731 [FREE Full text] [doi: 10.2147/PPA.S92108] [Medline: 26673985]

74. Dey V, Jones A, Spalding EM. Telehealth: acceptability, clinical interventions and quality of life in peritoneal dialysis. Sage Open Med 2016;4:2050312116670188 [FREE Full text] [doi: 10.1177/2050312116670188] [Medline: 27757228]

75. Ong SW, Jassal SV, Miller JA, Porter EC, Cafazzo JA, Seto E, et al. Integrating a smartphone-based self-management system into usual care of advanced CKD. Clin J Am Soc Nephrol 2016 Jun 6;11(6):1054-1062 [FREE Full text] [doi: 10.2215/CJN.10681015] [Medline: 27173169]

76. Hayashi A, Yamaguchi S, Waki K, Fujiu K, Hanafusa N, Nishi T, et al. Testing the feasibility and usability of a novel smartphone-based self-management support system for dialysis patients: a pilot study. JMIR Res Protoc 2017 Apr 20;6(4):e63 [FREE Full text] [doi: 10.2196/resprot.7105] [Medline: 28428168] 
77. Liu N, Kim J, Jung Y, Arisy A, Nicdao MA, Mikaheal M, et al. Remote monitoring systems for chronic patients on home hemodialysis: field test of a copresence-enhanced design. JMIR Hum Factors 2017 Aug 29;4(3):e21 [FREE Full text] [doi: 10.2196/humanfactors.7078] [Medline: 28851680]

78. Elbert NJ, van Os-Medendorp H, van Renselaar W, Ekeland AG, Hakkaart-van Roijen L, Raat H, et al. Effectiveness and cost-effectiveness of ehealth interventions in somatic diseases: a systematic review of systematic reviews and meta-analyses. J Med Internet Res 2014 Apr 16;16(4):e110 [FREE Full text] [doi: 10.2196/jmir.2790] [Medline: 24739471]

79. Tsai YC, Hung CC, Hwang SJ, Wang SL, Hsiao SM, Lin MY, et al. Quality of life predicts risks of end-stage renal disease and mortality in patients with chronic kidney disease. Nephrol Dial Transplant 2010 May;25(5):1621-1626. [doi: 10.1093/ndt/gfp671] [Medline: 20037172]

80. Mapes DL, Lopes AA, Satayathum S, McCullough KP, Goodkin DA, Locatelli F, et al. Health-related quality of life as a predictor of mortality and hospitalization: the dialysis outcomes and practice patterns study (DOPPS). Kidney Int 2003 Jul;64(1):339-349 [FREE Full text] [doi: 10.1046/j.1523-1755.2003.00072.x] [Medline: 12787427]

81. Kelders SM, Kok RN, Ossebaard HC, van Gemert-Pijnen JE. Persuasive system design does matter: a systematic review of adherence to web-based interventions. J Med Internet Res 2012 Nov 14;14(6):e152 [FREE Full text] [doi: 10.2196/jmir.2104] [Medline: 23151820]

82. Michie S, Yardley L, West R, Patrick K, Greaves F. Developing and evaluating digital interventions to promote behavior change in health and health care: recommendations resulting from an international workshop. J Med Internet Res 2017 Jun 29;19(6):e232 [FREE Full text] [doi: 10.2196/jmir.7126] [Medline: 28663162]

83. Wade DT. Research into the black box of rehabilitation: the risks of a type III error. Clin Rehabil 2001 Feb;15(1):1-4. [doi: 10.1191/026921501675961253] [Medline: 11237155]

84. van der Meij E, Anema JR, Leclercq WK, Bongers MY, Consten EC, Koops SE, et al. Personalised perioperative care by e-health after intermediate-grade abdominal surgery: a multicentre, single-blind, randomised, placebo-controlled trial. Lancet 2018 Jul 7;392(10141):51-59. [doi: 10.1016/S0140-6736(18)31113-9] [Medline: 29937195]

85. Chen J, Ou L, Hollis SJ. A systematic review of the impact of routine collection of patient reported outcome measures on patients, providers and health organisations in an oncologic setting. BMC Health Serv Res 2013 Jun 11;13:211 [FREE Full text] [doi: 10.1186/1472-6963-13-211] [Medline: 23758898]

86. Jonkman NH, Westland H, Groenwold RH, Ågren S, Anguita M, Blue L, et al. What are effective program characteristics of self-management interventions in patients with heart failure? An individual patient data meta-analysis. J Card Fail 2016 Nov;22(11):861-871 [FREE Full text] [doi: 10.1016/j.cardfail.2016.06.422] [Medline: 27374838]

87. Bos-Touwen I, Jonkman N, Westland H, Schuurmans M, Rutten F, de Wit N, et al. Tailoring of self-management interventions in patients with heart failure. Curr Heart Fail Rep 2015 Jun;12(3):223-235 [FREE Full text] [doi: 10.1007/s11897-015-0259-3] [Medline: $\underline{25929690]}$

88. Duff OM, Walsh DM, Furlong BA, O'Connor NE, Moran KA, Woods CB. Behavior change techniques in physical activity ehealth interventions for people with cardiovascular disease: systematic review. J Med Internet Res 2017 Aug 2;19(8):e281 [FREE Full text] [doi: 10.2196/jmir.7782] [Medline: 28768610]

89. van Velsen L, Wentzel J, van Gemert-Pijnen JE. Designing ehealth that matters via a multidisciplinary requirements development approach. JMIR Res Protoc 2013 Jun 24;2(1):e21 [FREE Full text] [doi: 10.2196/resprot.2547] [Medline: 23796508]

90. Fleuren MA, Paulussen TG, van Dommelen P, van Buuren S. Towards a measurement instrument for determinants of innovations. Int J Qual Health Care 2014 Oct;26(5):501-510 [FREE Full text] [doi: 10.1093/intqhc/mzu060] [Medline: 24951511]

91. Huijg JM, Gebhardt WA, Dusseldorp E, Verheijden MW, van der Zouwe N, Middelkoop BJ, et al. Measuring determinants of implementation behavior: psychometric properties of a questionnaire based on the theoretical domains framework. Implement Sci 2014 Mar 19;9:33 [FREE Full text] [doi: 10.1186/1748-5908-9-33] [Medline: 24641907]

92. Verberne WR, Das-Gupta Z, Allegretti AS, Bart HA, van Biesen W, García-García G, et al. Development of an international standard set of value-based outcome measures for patients with chronic kidney disease: a report of the international consortium for health outcomes measurement (ICHOM) CKD working group. Am J Kidney Dis 2019 Mar;73(3):372-384 [FREE Full text] [doi: 10.1053/j.ajkd.2018.10.007] [Medline: 30579710]

93. Eysenbach G, Consort-eHealth Group. Consort-eHealth: improving and standardizing evaluation reports of Web-based and mobile health interventions. J Med Internet Res 2011 Dec 31;13(4):e126 [FREE Full text] [doi: 10.2196/jmir.1923] [Medline: 22209829]

94. Liu L, Stroulia E, Nikolaidis I, Miguel-Cruz A, Rios Rincon A. Smart homes and home health monitoring technologies for older adults: a systematic review. Int J Med Inform 2016 Jul;91:44-59. [doi: 10.1016/j.ijmedinf.2016.04.007] [Medline: 27185508]

95. Capurro D, Ganzinger M, Perez-Lu J, Knaup P. Effectiveness of ehealth interventions and information needs in palliative care: a systematic literature review. J Med Internet Res 2014 Mar 7;16(3):e72 [FREE Full text] [doi: 10.2196/jmir.2812] [Medline: 24610324] 
96. Fisher E, Law E, Palermo TM, Eccleston C. Psychological therapies (remotely delivered) for the management of chronic and recurrent pain in children and adolescents. Cochrane Database Syst Rev 2015 Mar 23(3):CD011118 [FREE Full text] [doi: 10.1002/14651858.CD011118.pub2] [Medline: 25803793]

97. Chen YR, Schulz PJ. The effect of information communication technology interventions on reducing social isolation in the elderly: a systematic review. J Med Internet Res 2016 Jan 28;18(1):e18 [FREE Full text] [doi: 10.2196/jmir.4596] [Medline: 26822073]

98. Rost T, Stein J, Löbner M, Kersting A, Luck-Sikorski C, Riedel-Heller SG. User acceptance of computerized cognitive behavioral therapy for depression: systematic review. J Med Internet Res 2017 Sep 13;19(9):e309 [FREE Full text] [doi: 10.2196/jmir.7662] [Medline: 28903893]

\author{
Abbreviations \\ BP: blood pressure \\ CCAT: Crowe Critical Appraisal Tool \\ CKD: chronic kidney disease \\ CRD: Centre for Reviews and Dissemination \\ eHealth: electronic health \\ GFR: glomerular filtration rate \\ NCDs: noncommunicable diseases \\ PRISMA: Preferred Reporting Items for Systematic Reviews and Meta-Analyses \\ QoL: quality of life \\ RCT: randomized controlled trial
}

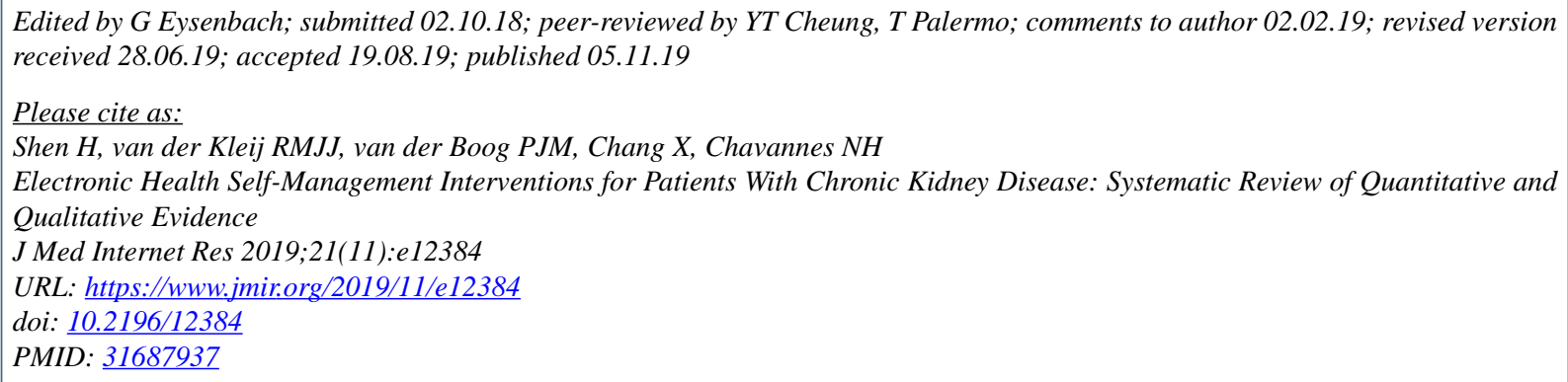

CHongxia Shen, Rianne MJJ van der Kleij, Paul JM van der Boog, Xinwei Chang, Niels H Chavannes. Originally published in the Journal of Medical Internet Research (http://www.jmir.org), 05.11.2019. This is an open-access article distributed under the terms of the Creative Commons Attribution License (https://creativecommons.org/licenses/by/4.0/), which permits unrestricted use, distribution, and reproduction in any medium, provided the original work, first published in the Journal of Medical Internet Research, is properly cited. The complete bibliographic information, a link to the original publication on http://www.jmir.org/, as well as this copyright and license information must be included. 